Int. J. Dev. Biol. 53: 19-27 (2009)

doi: $10.1387 / \mathrm{ijdb} .082618 \mathrm{ss}$

\title{
Organelles in developing neurons: essential regulators of neuronal morphogenesis and function
}

\author{
SAYAKA SEKINE ${ }^{1}$, MASAYUKI MIURA ${ }^{1,2}$ and TAKAHIRO CHIHARA*,1,2 \\ ${ }^{1}$ Department of Genetics, Graduate School of Pharmaceutical Sciences, University of Tokyo and ${ }^{2}$ CREST, JST, Tokyo, Japan
}

\begin{abstract}
Eukaryotic cells contain multiple intracellular organelles which are structurally and functionally distinct membrane-delimited compartments. Organelles play vital roles in many cellular events in essentially all eukaryotic cells. Although the canonical roles of organelles are well described by classical in vitro studies, little is known about the specific physiological roles of organelles in neurons, which possess extremely polarized cellular structures and have a massive cellular volume compared with most eukaryotic cells. Studies that make use of recently developed genetic and microscopic techniques are currently elucidating the unexpectedly specialized roles of intracellular, membrane-delimited organelles in neuronal morphogenesis and function, and in human disease. Here we review recent advances in understanding the roles of organelles (the ERGolgi secretory pathway, endosomes and mitochondria) in developing neurons.
\end{abstract}

KEY WORDS: developing neuron, organelle, secretory pathway, endosome, mitochondria

\section{Introduction}

Eukaryotic cells contain multiple intracellular compartments: organelles that are structurally and functionally distinct and have a delimiting membrane. Because organelles play vital roles in numerous cellular events, including cell proliferation, differentiation, maintenance, and death, it is essential to understand their specific roles in different cell types. Classically, the structures and functions of organelles have been studied intensively in non-neuronal cells, such as cultured epithelial cell lines, but little is known about organelles' functions in developing and mature neurons, which possess extremely polarized cellular structures (i.e., cell body, axon, and dendrites), whose cellular volume can exceed that of most eukaryotic cells by as much as 10,000-fold, and most of whose cellular volume (axoplasm) resides at a great distance from their nucleus.

It is currently unclear if the various organelles play the same roles in neurons as in non-neuronal cells, although evidence for specific functions is beginning to accumulate. Newly developed genetic and microscopic techniques (Venken and Bellen, 2005, Miyawaki, 2005) have permitted investigations into physiological and specialized functions of organelles in neurons that are ordinarily masked by their pleiotropic functions. For instance, by using the genetic mosaic method in Drosophila, one can manipulate the genes regulating organelles' functions in individual neurons of the intact brain, which permits investigation of the physiological roles of organelles in neurons in vivo (Lee and Luo, 1999). Other methodologies than genetic mosaicism are technically much harder to use for this purpose, because disruptions in the in vivo physiological functions of organelles (i.e., by genetic manipulation) are typically lethal to the organism. Recent studies using these new genetic and microscopic techniques have revealed unexpected and specific roles of organelles in neuronal morphogenesis and function, and in human disease.

Here, we review recent advances in understanding the functions of organelles in developing and mature neurons. Note that, in addition to the membrane-delimited organelles (endoplasmic reticulum (ER)-Golgi secretory pathway, endosomes, and mitochondria) reviewed here, the centrosome is known to have specific roles in neurons. For a comprehensive review of centrosome function in neuronal development, see Higginbotham and Gleeson (2007).

\section{Secretory pathway}

The ER and the Golgi apparatus are the essential organelles for

\footnotetext{
Abbreviations used in this paper: ALS, amyotrophic lateral sclerosis; CICR, Ca2+-induced Ca2+ release; DA, dendritic arborization; DRG, dorsal route ganglia; ER, endoplasmic reticulum; ESCRT-III, endosomal sorting complex required for transport; KHC, kinesin heavy chain; LTP, long-term potentiation; MVBs, multivesicular bodies; NGF, nerve growth factor; PI3kinase, phosphoinositide 3-kinase; TGN, trans-Golgi network.
}

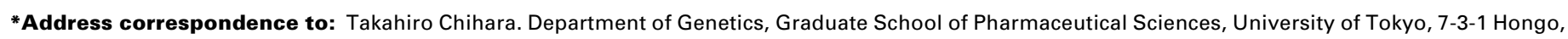
Bunkyo-ku, Tokyo 113-0033, Japan. Fax: +81-3-5841-4867. e-mail: tchihara@mol.f.u-tokyo.ac.jp

Accepted: 6 June 2008. Published online: 5 December 2008. 


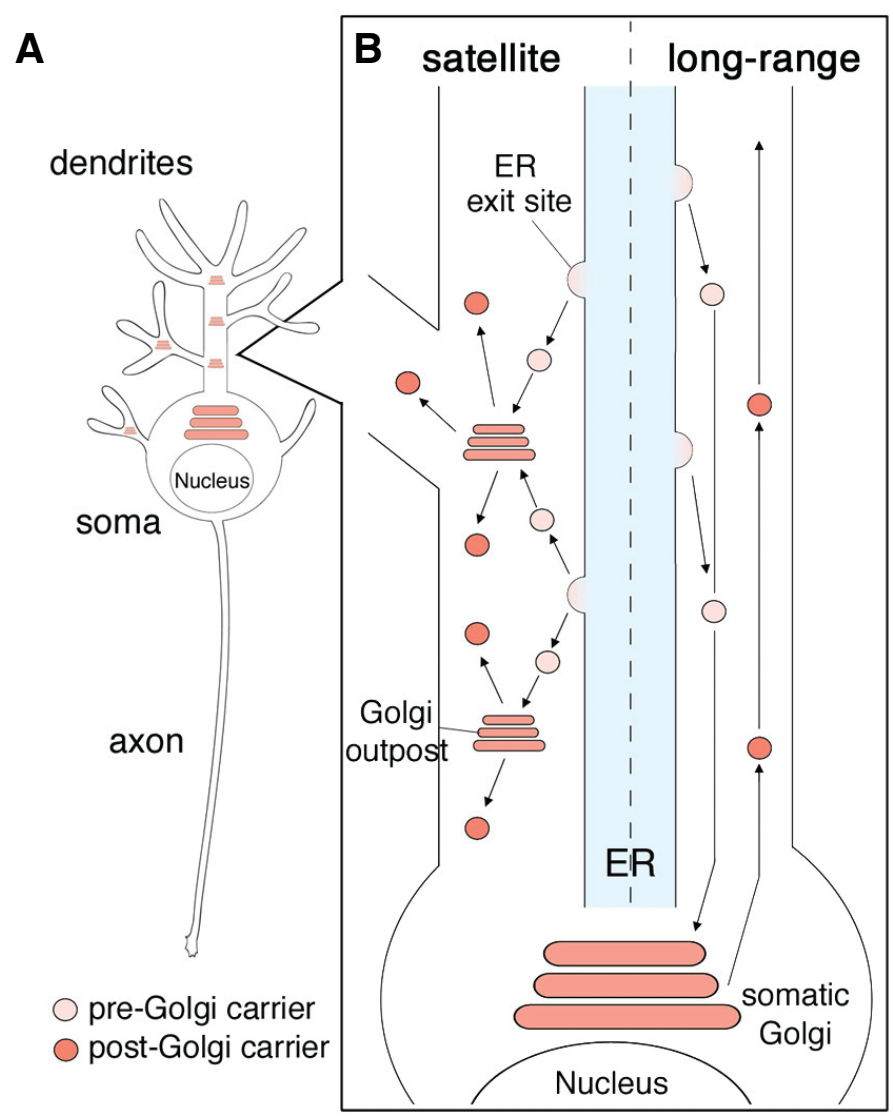

Fig. 1. Secretory pathways in neurons. (A) A schematic drawing representing the localization of somatic Golgi and dendritic Golgi outposts in a neuron. (B) Dual modes of secretory pathways in neurons. Both modes of ER-Golgi transport occur in parallel in the dendrites. The left half of the dendrite shows a schematic drawing of the "Satellite" secretory pathway. The cargo buds from the ER exit sites and traffics to adjacent Golgi outposts. The right half of the dendrite shows the "Longrange" secretory pathway. The pre-Golgi carriers bud from the dendritic $E R$ exit sites and traffic in the retrograde direction toward the somatic Golgi apparatus.

the synthesis and processing of proteins and lipids. Proteins destined for secretion and membrane localization are synthesized and posttranslationally modified, including glycosylation and disulfide-bond formation, in the ER. The modified cargo is transported to the Golgi apparatus for further modifications and finally to the appropriate target via the trans-Golgi network (TGN). This serial pathway, referred to as the secretory pathway, is not only biogenic but also plays other important roles in the cells of multicellular organisms. For instance, epithelial cells that exhibit polarized secretion have a polarized secretory pathway, and their Golgi apparatus is directed toward the secretory surface. In neurons, recent studies have revealed the secretory pathways to be uniquely specialized for functions related to neuronal morphogenesis and polarization (Horton and Ehlers, 2003a, Horton et al., 2005, Ye et al., 2007).

\section{Spatial organization of the ER and Golgi}

The ER is a continuous endomembrane that extends throughout the cell, functioning as both the site of synthesis for membrane proteins and as an intracellular compartment for calcium signaling. As in non-neuronal cells, the neuronal ER is present throughout the soma, axons, and dendrites, with sporadic "ER exit sites" where the ER cargo is concentrated into vesicles that are transported to the Golgi apparatus (Fig. 1) (Terasaki et al., 1994, Horton and Ehlers, 2003a).

The Golgi apparatus consists of organized stacks of disc-like compartments that receive proteins and lipids from the ER and dispatch them to a variety of destinations. In addition to the Golgi apparatus, which inhabits the perinuclear regions of the soma, neurons have small Golgi-like compartments termed "Golgi outposts" in their dendrites (Fig. 1) (Horton and Ehlers, 2003a, Torre and Steward, 1996). In cultured rat hippocampal neurons, the Golgi outposts are most common in the proximal dendrites, but they are also present more distally in secondary and tertiary dendrites, and have not been found in axons (Horton and Ehlers, 2003a, Horton et al., 2005). Similarly, the Golgi outposts are reported to reside predominantly in the dendrites of Drosophila dendritic arborization (DA) neurons, suggesting that the subcellular localization of Golgi outposts is evolutionary conserved, and thus that the Golgi outposts in dendrites play biologically significant roles (Ye et al., 2007). However, it is still unclear how Golgi outposts are produced, selectively transported to dendrites, and largely excluded from axons.

\section{Dual modes of neuronal secretory pathways}

One possible implication of the special arrangement in dendrites is that the ER exit site and Golgi outposts actually comprise local secretory pathways. Horton and Ehlers addressed this question in cultured rat hippocampal neurons by utilizing live-cell imaging of GFP-tagged cargo proteins (Horton and Ehlers, 2003a). They demonstrated that newly synthesized membrane proteins concentrate at the ER exit sites throughout the dendrites, from which they bud into highly mobile carriers. These carriers then traffic either long distances to fuse with the somatic Golgi apparatus or locally to fuse with the dendritic Golgi outposts, which produce both integral membrane proteins and the neuronal growth factor BDNF. From these observations, Horton and Ehlers proposed the existence of dual modes of secretory pathway in neurons (Fig. 1). In their model, one mode, the "long-range" mode, involves cargo from dendritic ER exit sites traveling in retrograde toward the somatic Golgi apparatus. In the other, the "satellite (local)" mode, the action is restricted to the dendrites, in which cargo travel from dendritic ER exit sites to dendritic Golgi outposts. The presence of a satellite secretory pathway supports the possibility that local protein translation and modification occur at the dendrites, which is also implied by the existence of mRNAs in the dendrites (Steward and Schuman, 2003). For what are Golgi outposts required? It might be possible that Golgi outposts are required for the rapid response to the environmental changes in the peripheral dendrites as it was hypothesized as a requirement of local protein translation (Steward and Schuman, 2003). It is interesting to see the effect of disrupting Golgi outposts as demonstrated by $\mathrm{Ye}$ et al. (2007) on local protein translation.

Interestingly, however, some neurons have no Golgi outposts and depend on the somatic Golgi apparatus entirely, and even in neurons possessing dendritic Golgi outposts, the bulk of the ERGolgi traffic is in the long-range pathway (Horton and Ehlers, 2003a). What is the difference between neurons with and without 
Golgi outposts? Are Golgi outposts required in neurons with long and/or highly elaborated dendrites as those dendritic terminals should be distant from somatic ER-Golgi secretory pathway? It will be important for future studies to investigate the neuronal morphologies with or without Golgi outposts, and the functional differences between the "long-range" and "satellite" secretory pathways, and to determine the types of cargo targeted to the satellite pathway rather than to the long-range pathway in neurons.

\section{Secretory pathways in neuronal morphogenesis and polar- ization}

The neuronal secretory pathway seems to have active roles in neuronal morphogenesis. In rat hippocampal pyramidal neurons, the somatic Golgi apparatus tends to be polarized toward longer dendrites, but not toward the shorter ones (Fig. 1) (Horton et al., 2005). This asymmetric distribution of the somatic Golgi apparatus precedes the appearance of dendritic asymmetry, suggesting the potential involvement of the somatic Golgi in dendritic growth. In addition, dendrites harboring Golgi outposts have longer and more complex morphologies than those without. The importance of secretory pathways in dendritic morphology was experimentally proven by disassembling the Golgi apparatus with brefeldin $A$ and by blocking late secretory trafficking with a kinase-dead (KD) mutant of protein kinase D1 (PKD-KD) (Horton and Ehlers, 2003a). Cultured rat neurons treated with brefeldin $A$ and mouse neurons expressing PKD-KD showed significantly reduced dendritic outgrowth and maintenance, suggesting that the secretory pathway in neurons plays an essential role in the development and maintenance of the dendritic morphology.

A study of Drosophila dendritic arborization (DA) neurons provided in vivo evidence for the importance of the neuronal secretory pathway during development. From a genetic screen to identify mutants affecting the dendritic morphology of DA neurons, Ye et al. found several mutants that shared a common phenotype of reduced dendritic arbors but normal axon growth (Ye et al., 2007). Strikingly, three of these mutations were in genes for critical regulators of the secretory pathway (Drosophila homologs of Sec23, Sar1, and Rab1). Mosaic analyses revealed that these genes function cellautonomously to regulate dendritic, but not axonal, growth in vivo.

Specific evidence for the significance of the dendritic Golgi outposts was obtained by damaging them with an intense pulse of laser (Ye et al., 2007). The damage affected the dynamics of dendritic development, in which the dendritic branches neither extended nor retracted normally. Thus, Golgi outposts may serve as mobile stations for membrane traffic to dendrites but not to axons, and contribute to the regulation of morphological changes in dendritic arbors. Such regulation might involve both genetic programs for specifying intrinsic differences in the dendritic patterning of different neurons and activity-dependant modifications of dendritic arbors.

\section{Involvement of TGN in axon guidance}

After the appropriate posttranslational modifications are carried out in the ER and the Golgi apparatus, cargo is transported to appropriate targets, such as the plasma membrane or lysosome (Rodriguez-Boulan et al., 2005). This cargo-sorting system is called the trans-Golgi network (TGN). Studies on Commissureless (Comm), a Drosophila late endosomal protein, revealed an unexpectedly specific role of the TGN in axon guidance (Keleman et al., 2002, Keleman et al., 2005). During the development of the Drosophila embryonic nerve cord, many commissural neurons extend their axons across the midline to form the commissure and to reach their final targets on the opposite (contralateral) side. The ipsilateral neurons extend axons along the same side as the cell body and never cross the midline. This decision, to cross or not to cross the midline, is temporally regulated by the expression of Comm in the commissural neurons.

Comm acts as a sorting receptor for Roundabout (Robo), a receptor that responds to a repulsive ligand, Slit, which is expressed in the midline glial cells (Dickson, 2002). In ipsilateral neurons, Robo is expressed and properly integrated into the plasma membrane, which directs the axonal path not to cross the midline by recognizing the hypothetical gradient of Slit ligand (Fig. 2A). In contrast, in the commissural neurons, Comm is expressed and forms a complex with the Robo receptor, which redirects Robo from its site of synthetic (the ER-Golgi) to the lysosomal degradation pathway. Eventually, this results in little or no Robo expression on the plasma membrane of the commissural neu-

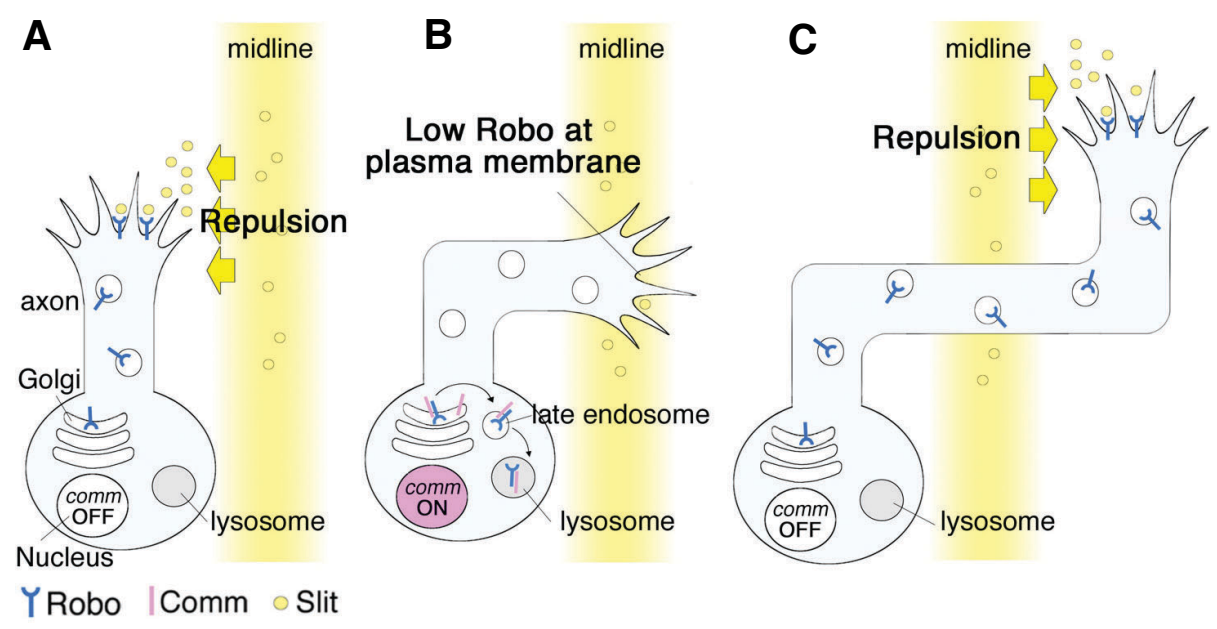

Fig. 2. Model for the regulation of axon midline crossing by Commissureless (Comm). (A) Both ipsilateral and contralateral neurons initially express the Robo protein, which is inserted into the plasma membrane of the growth cones. This allows axons to sense Slit and avoid the midline. (B) Comm is specifically expressed by the contralateral neurons, while their axons are crossing the midline. Comm diverts Robo in the trans-Golgi network to the endosome/lysosomal degradation pathway, and both Robo and Comm are degraded in the lysosome. Comm expression therefore results in the loss of Robo from the plasma membrane. (C) After the midline crossing is completed, Comm expression is turned off, which restores Robo trafficking to the plasma membrane. Robo at the growth cones can recognize and be repelled by Slit at the midline, which prevents the axon from recrossing the midline. 
rons, which cannot sense Slit without it, and therefore cross the midline (Fig. 2B). Interestingly, once the commissural axons cross the midline, Comm expression is turned off, "trapping" the growing tip of the axon on the side of the embryo contralateral to the neuronal cell body (Fig. 2C). The spatiotemporal change of Comm expression is an example of a novel regulatory mechanism for axon guidance receptors, which are indeed utilized to create the neural network of Drosophila. To understand more about how axon guidance is determined, it will be important to elucidate the molecular mechanisms underlying the spatiotemporal degradation of other neuronal proteins involved in morphogenesis or axon pathfinding via the TGN-lysosome pathway. Whether or not the regulatory morphogenic mechanisms that are used in Drosophila are shared with mammalian neuronal development is an exciting question for the near future.

In addition to the decision "degrade, or not to degrade" by Comm protein described above, the destination of neuronal proteins (and membrane) seems to be further finely tuned in order to make axonal growth cones properly turn. Tojima et al. recently reported that the attractive, but not repulsive axon guidance needs directional exocytosis to the side of axonal turning, which is regulated by asymmetric elevation of the $\mathrm{Ca}^{2+}$ concentration accompanied by $\mathrm{Ca}^{2+}$-induced $\mathrm{Ca}^{2+}$ release $(\mathrm{CICR})$ in the growth cone (Tojima et al., 2007). Interestingly, the $\mathrm{Ca}^{2+}$ signals without $\mathrm{CICR}$ had no effect on exocytosis. Therefore, it is of great interest to examine why and how $\mathrm{Ca}^{2+}$ signals with $\mathrm{CICR}$ specifically induces exocytosis in the attractive axon.

\section{Endosomes}

The intracellular membranous structures called endosomes are key compartments of endocytosis-related vesicular trafficking (Kennedy and Ehlers, 2006, Rodriguez-Boulan et al., 2005). Endosomes are roughly classified into four types: the early endosome, late endosome, recycling endosome, and lysosome. From numerous studies in non-neuronal cells, the essential roles of endosomal trafficking in protein sorting, recycling, and degradation have been well described. Endocytosed vesicles first fuse with early endosomes, which receive plasma membrane and membrane proteins directly. These early endosomes act as sorting stations that direct cargos to the late endosome/lysosomal pathway for degradation or to recycling endosomes, which return membrane phospholipids and/or membrane proteins to the plasma membrane. In addition, trafficking through early endosomes is essentially linked with the sending, receiving, and transduction of intracellular signaling during development. Despite these welldescribed essential functions of the endosomal system, its specific roles in neuronal morphogenesis are just beginning to be learned.

\section{Localization of endosomes in neurons}

To understand the roles of endosomes in neurons, an obvious first question is where and how the endosomes are distributed in them. Internal membranous structures resembling endosomes have been described in dendrites, and even in dendritic spines (Prekeris et al., 1999, Cooney et al., 2002, Park et al., 2004, Park et al., 2006). These dendritic endosomes seem to be involved in recycling neurotransmitter receptors (Brown etal., 2007, Maxfield and McGraw, 2004, Park et al., 2004) and in dendritic spine morphogenesis (Horton and Ehlers, 2003b, Park et al., 2006).

In axons, although endosomes are not as abundant as in the somato-dendritic domain, they indeed exist. By taking advantage of the feature that endosomes possess low $\mathrm{pH}$, Overly and Hollenbeck used a ratiometric $\mathrm{pH}$ indicator to examine the spatial and dynamic organization of the endosomal system in the axons of cultured sympathetic neurons. They reported that not only do axonal endosomes exist, but that their distribution is surprisingly unequal along the axon (Overly and Hollenbeck, 1996). For instance, about half of the endosomes in the growth cone exhibit a $\mathrm{pH}$ value characteristic of early $(\mathrm{pH} 5.9-6.2)$ and recycling $(\mathrm{pH}$ 6.4-6.5) endosomes, whereas only $4 \%$ of the axonal shaft endosomes have $\mathrm{pH}$ values in this range. This unique distribution of endosomes in the axon may imply that early/recycling endosomes in the growth cone have essential roles in growth cone functions, such as neurite extension.

\section{Endosomes in neurite extension}

Neurite extension is initiated and promoted by the activation of the nerve growth factor (NGF) signaling pathway in PC12 cells, a rat pheochromocytoma cell line. NGF binds to its TrkA receptor at the plasma membrane, leading to "transient" activation of the Ras-ERK signaling pathway and phosphoinositide 3 (PI3) -kinase, which promotes cell survival (Fig. 3) (Moises et al., 2007, Liu et al., 2007). Next, the NGF-TrkA complex is endocytosed and localized to early endosomes. The early endosomes that contain the NGF-TrkA complex are called "signaling endosomes" and are thought to be the site of activation for the small GTPase Rap1. TrkA is then transferred to late endosomes, from which it maintains the sustained activation of the Rap1-ERK signaling pathway (Hisata et al., 2007). Because these "transient" and "sustained" activations of the NGF signal occur in endosomes, the coordinated intracellular dynamics between early endosomes (including signaling endosomes) and late endosomes containing the NGF-TrkA complex are crucial for regulating the intracellular signals required for neurite extension.

During neurite extension, membrane is added efficiently at the growth cone (Craig et al., 1995), and the inhibition of membrane recycling by recycling endosomes prevents neurite extension (Schmidt and Haucke, 2007). Striking evidence for this contribution of recycling endosomes was obtained with the identification of Protrudin, an evolutionarily conserved Rab11-binding protein (Shirane and Nakayama, 2006). In PC12 cells, both neurite extension and the phosphorylation of Protrudin are induced by NGF stimulation, which causes Protrudin to bind to Rab11, a small GTPase that regulates membrane recycling to the plasma membrane (Fig. 3). The overexpression of Protrudin induces neurite-like structures through its binding to Rab11, even in nonneuronal cells, such as HeLa cells, whereas the knockdown of Protrudin in PC12 cells inhibits NGF-induced neurite formation and induces membrane spreading in all directions. These results indicate that the Protrudin-Rab11 system has essential and instructive roles in the directional membrane recycling required for neurite extension. It will be interesting to learn how the Protrudin-Rab11 system recognizes the direction in which neurite extension should be promoted.

\section{Endosomes in dendrite development and plasticity}

The degradation pathway via the late endosomes and lysos- 
omes also seems to be involved in neuronal morphogenesis. A recent genetic study using Drosophila DA sensory neurons provided in vivo evidence that the endosomal pathway to lysosomal degradation is critical for dendrite development (Sweeney et al., 2006). Shrub is a Drosophila homolog of yeast Snf7, a key component of ESCRT-III (endosomal sorting complex required for transport) and is involved in the trafficking of membrane proteins to the lysosomes via multivesicular bodies (MVBs). Mutant DA neurons for shrub gene generated in the Drosophila larva exhibited increased number of dendritic branching cellautonomously. This observation suggests that protein degradation in the lysosomal pathway must be properly regulated for appropriate dendritic branching to occur. The next question to be addressed is what component must be degraded in the lysosomes for the appropriate regulation of dendritic branching. In addition, to understand the regulatory roles of the late endosome/ lysosomal pathway in dendrite development, it will be important to resolve whether the lysosomal degradation system is activated for all endocytosed proteins, or whether particular sets of membrane proteins regulating dendritic branching are specifically sorted into the lysosomal pathway.

The endosomal system is also involved in neuronal plasticity, the dynamic modification of synaptic strength. Long-term potentiation (LTP) of synaptic strength promotes the growth of dendritic spines, the tiny protrusions along dendrites. Dendritic spines are thought to be the dominant cellular structures for learning and memory, and their sizes are finely regulated by stimuli that induce LTP in hippocampal neurons. Using time-lapse imaging and electron microscopy, Park etal., showed that recycling endosomes are located within or at the base of dendritic spines in rat hippocampal slices (Park et al., 2006). Interestingly, LTP induced by the activation of synaptic NMDA receptors causes the rapid entrance of recycling endosomes into the spines, concurrent with the formation of new spines and the growth of existing spines. The expression of a dominant-negative form of Rab11, Rab11 S25N, blocks the LTP-induced growth of spines, suggesting a mechanistic link between the Rab11-mediated trafficking of recycling endosomes and the morphological plasticity of dendritic spines. In addition, recycling endosomes are reported to be essential for supplying postsynaptic AMPA-type glutamate receptors during LTP (Brown et al., 2007, Park et al., 2004). These studies suggest that the trafficking of recycling endosomes regulates the plasticity of dendritic spines both morphologically and functionally.

\section{Mitochondria}

Mitochondria are vital organelles present in all eukaryotic cells. In addition to their energy-generating function, they participate in metabolism, calcium signaling, and apoptosis (Chan, 2006, Kujoth et al., 2005). Reflecting their diverse functions, mitochondria take on various shapes that vary from vesicular to tubular network structures, via fission and fusion events. Given their well-studied functions, it might seem that one could easily predict the consequences of mitochondrial dysfunction. However, because mitochondria play so many essential roles in cell survival, differentiation, and apoptosis, the effects of mitochondrial dysfunction in multicellular organisms can be complex and difficult to anticipate. In addition, a number of inherited neurological diseases are owing to mutations of mitochondria-related genes, implying that mitochondria are likely to have specialized functions in neurons. Therefore, it is important to elucidate the roles of mitochondria in neuronal development, maintenance, and disease.

\section{Localization of mitochondria in neurons}

As a first step toward understanding the roles of mitochondria in neurons, their subcellular localizations need to be established, and considerable progress has been made in this area. Although the cell body is an obvious location for mitochondria, it seems likely that they would also be concentrated at sites where ATP consumption is high and/or $\mathrm{Ca}^{2+}$ buffering is needed, such as in the growth cone. Morris and Hollenbeck approached this fundamental question by labeling the mitochondria of living chick sympathetic neurons with vital dyes (4-di2-ASP or rhodamine 123), and reported that mitochondria were more abundant in the distal region of growing axons than in that of non-
Fig. 3. Endosomes in NGF-TrkA-induced neurite extension. In PC12 cells, NGF binds to its TrkA receptor at the plasma membrane, leading to first transient and then sustained activation of the ERK signal in the early endosomes (signaling endosomes) and late endosomes, respectively, and these signals are required for neurite growth. Phosphorylation of Protrudin, induced by the NGF-TrkA signaling, enhances Protrudin binding to Rab11, which regulates the direction of membrane recycling for neurite extension. 
growing axons (Morris and Hollenbeck, 1993). Live observation of mitochondrial behavior indicated that mitochondrial transport within the axon was bidirectional, moving to the distal tip (anterograde) and back to the cell body (retrograde), and that the net mitochondrial movement was anterograde in growing axons but retrograde in non-growing axons. These findings of skewed mitochondrial distributions and transport make sense in terms of growing axons having a greater energy (ATP) requirement than non-growing axons. How mitochondria are attracted to or accumulate in the distal region of the growing axon is a fascinating issue that remains to be studied.

In addition to describing the variable distributions of mitochondria in growing vs non-growing axons, Hollenbeck and colleagues studied the differences in mitochondrial activity between axons and dendrites (Overly et al., 1996). The mitochondrial energy status correlates with the mitochondrial membrane potential, which can be monitored with a vital mitochondrial dye, JC-1. When the $\mathrm{JC}-1$ concentration in mitochondria is low, it emits a green fluorescence $(527 \mathrm{~nm})$. When the mitochondrial membrane potential increases, more JC-1 dye accumulates in the mitochondria and forms "J-aggregates" that emit red fluorescence $(590 \mathrm{~nm})$. In cultured embryonic rat hippocampal neurons, after the axon and dendrites have matured, mitochondria are found in both the axon and dendrites. Interestingly, in these cells, mitochondria with J-aggregates are fairly numerous in the dendrites but are rare in the axon (except at the growth cone region), suggesting that the mitochondria in dendrites are metabolically more active than those in the axon. This observation has two interrelated and interesting implications. One is that local demands on the mitochondria could activate an increase in the mitochondrial membrane potential. The other is that dendrites may require more mitochondrial activity than axons do, except perhaps at the growing tip of axon.

\section{Mitochondria in dendrite maintenance and plasticity}

The biased distribution of mitochondrial activity (high in dendrites, low in axons) suggests that mitochondrial dysfunction could cause preferential defects in dendrites. Recent studies provide experimental evidence for mitochondria being required for dendrite development, maintenance, and plasticity. Drosophila olfactory projection neurons are a good model system for analyzing axon and dendrite morphologies, because individual projection neurons can be labeled and/or their genotype manipulated in vivo (in the brain) by using the genetic mosaic method (Jefferis et al., 2001, Lee and Luo, 1999) (Fig. 4). Projection neurons homozygous for a mutation in a gene that is essential for mitochondrial protein translation (e.g., a mitochondrial ribosome subunit or mitochondrial tRNA synthetases) show reduced dendritic elaborations but relatively normal axonal morphologies (Chihara et al., 2007) (Fig. 4C). Furthermore, these dendritic phenotypes are more severe in the mutant projection neurons of aged flies ( $>30$ days old), but the axons remain intact, suggesting that neuronal mitochondrial function is required in vivo for normal dendrite development and maintenance. This finding also suggests the potential differences in the sensitivity to mitochondrial dysfunction between dendrites and axons, and is indeed consistent with the differences of metabolic state of mitochondria between dendrites and axons, as described above. It might be interesting to examine the mitochondrial morphology and function in neurons defective in the polarity establishment, and vise versa.

Dendritic mitochondria also have essential roles in dendritic spine morphogenesis and plasticity. In cultured rat hippocampal neurons, the number of dendritic spines with mitochondria increases when the dendritic spines are actively growing (11 14 days in vitro), or when the neurons are subjected to repeated depolarization by $\mathrm{KCl}$ stimulation ( $\mathrm{Li}$ et al., 2004). Furthermore, the expression of dominant-negative forms of Drp1 or OPA1 (large GTPases that regulate mitochondrial morphology and distribution) reduces the dendritic mitochondrial content, leading to a loss of dendritic spines. On the other hand, stimulating mitochondrial respiration by treating neurons with creatine increases the number and plasticity of dendritic spines. These results indicate that mitochondria are important in dendritic spine morphogenesis and plasticity, and imply that the dynamic distribution of mitochondria relative to the dendritic spines could reflect the state of neuronal plasticity and of local ATP consumption.

\section{Axonal transport of mitochondria}

Mitochondria are distributed throughout the neuron, including the cell body, dendrite, and axon. In particular, mitochondria in the presynaptic region of an axon terminal are unequivocally important for local ATP production and $\mathrm{Ca}^{2+}$ buffering (Verstreken et al., 2005). Therefore, the localization of mitochondria to the tip of the axon, which is the most distant point from the cell body, is particularly crucial for proper neurotransmission. A recent series of studies in Drosophila revealed the molecular mechanisms of mitochondrial transport to presynaptic sites. milton was originally isolated as a mutant showing aberrant synaptic transmission and a loss of mitochondria from the axon terminal (Stowers et al., 2002). Milton is an evolutionarily conserved protein that forms a complex with kinesin heavy chain (KHC) and Miro, an atypical mitochondrial GTPase (Glater et al., 2006, Guo et al., 2005, Verstreken et al., 2005). This protein complex acts as a molecular link between mitochondria and microtubules, and is essential for the anterograde transport of mitochondria to the presynaptic site. Whether this protein complex also functions in the retrograde transport of mitochondria remains to be determined, as do the molecular details involved in the dissociation of mitochondria from the complex.

Recently, investigations have been made into the effects of activating the NGF/TrkA/PI3-kinase signaling pathway and of the actin cytoskeletal organization on mitochondrial transport in the axon (Chada and Hollenbeck, 2003, Chada and Hollenbeck, 2004). Chada and Hollenbeck reported that, in cultured neurons from chicken dorsal route ganglia (DRG), local stimulation of the axon shaft with NGF-coated beads causes local mitochondrial accumulation, and this NGF effect on mitochondrial behavior is disrupted by treatment with inhibitors of PI3-kinase (Chada and Hollenbeck, 2003, Chada and Hollenbeck, 2004). Interestingly, latrunculin B (an inhibitor of actin polymerization) does not affect the bidirectional movement of mitochondria, but strongly inhibits their NGF-induced accumulation, suggesting that the organization of the actin cytoskeleton is important for the local positioning of mitochondria in the axon. Given this finding, it will be particularly interesting to understand how the KHC-Milton-Miro complex, NGF signaling, and actin cytoskel- 
Fig. 4. Mitochondrial dysfunction in Drosophila olfactory projection neurons causes progressive defects in dendritic, but not axonal, terminals. (A) A scheme representing the dendritic and axonal projections of wildtype Drosophila olfactory projection neuron (PN). The antennal lobe ( $A L)$ consists of $\sim 50$ glomeruli, round shape synaptic structures. In general, PN dendrites target a single glomerulus, and $P N$ axon innervates lateral horn (LH). (B) A scheme representing the shape of Drosophila brain and relative positions of the AL, LH and optic lobe (OL). The AL is surrounded by $\sim 150$ PN cell bodies (green dots) in each hemisphere. PN dendrites and axons are not shown. Dotted rectangle represents the region of brain shown in (A). (C) Axons (a-c) and dendrites $\left(d-f^{\prime}\right)$ of wild-type $\left(a, d, d^{\prime}\right)$ and $\mathrm{tko}^{3 / 3}\left(b, c, e, f, e^{\prime}, f^{\prime}\right)$ PN single-cell clones generated by genetic mosaic method, MARCM system (Lee and Luo, 1999) in 2-5 days-old (a,b, d,e, $\left.d^{\prime}, e^{\prime}\right)$ and 30 days-old animal $\left(c, f, f^{\prime}\right)$. PN clones

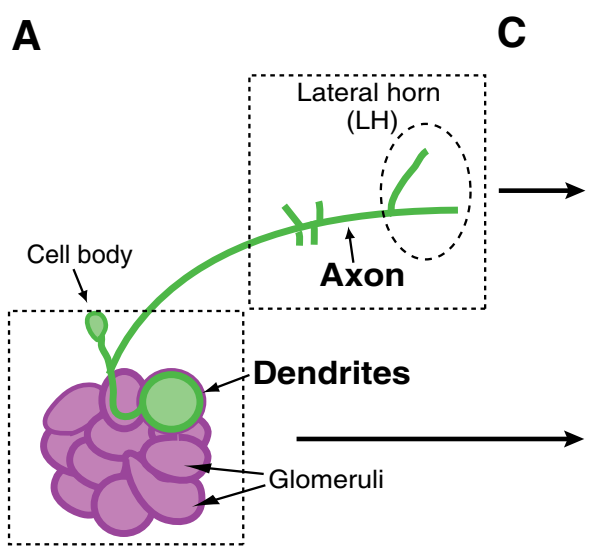

Antennal lobe $(\mathrm{AL})$

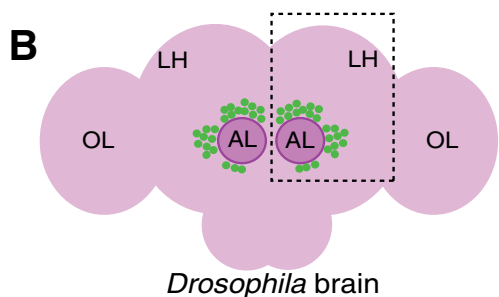

Wild-type PN
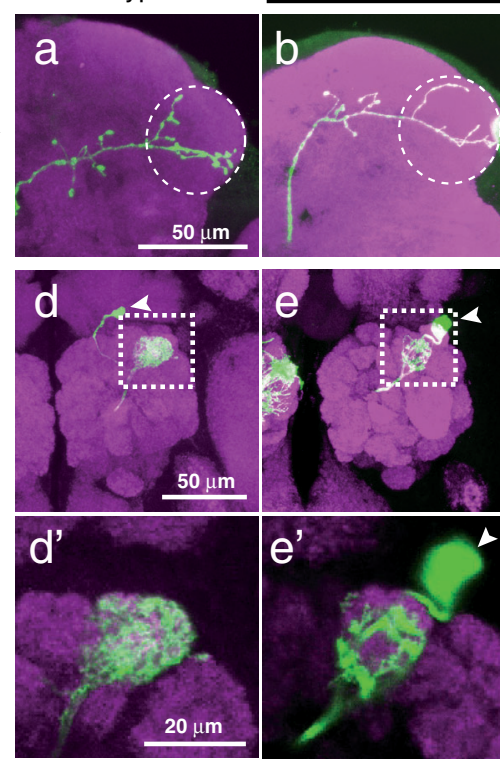

2-5 days old

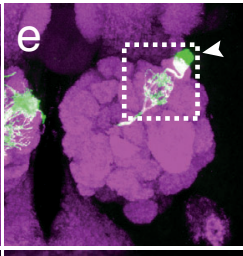

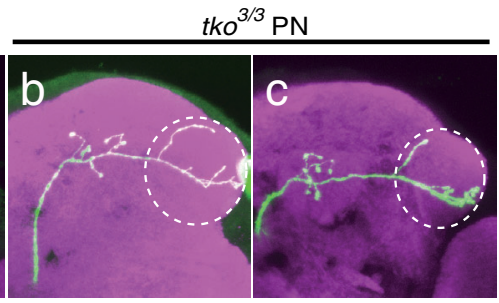
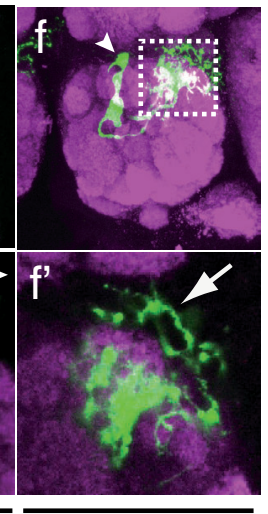

$\sim 30$ days old

and presynaptic regions are shown in green and magenta, respectively. Dotted circles in (a-c) indicate the region of lateral horn (LH). ( $\left.d^{\prime}, e^{\prime}, f^{\prime}\right)$ The enlarged images of dotted squares in $(d, e, f)$, respectively. Arrowheads in $\left(d, e, f, e^{\prime}\right)$ indicate PN cell bodies. tko is the null allele of technical knockout (tko) gene that encodes mitochondrial ribosome S12. Note that neuronal morphologies (axon and dendrites) of tko ${ }^{3 / 3}$ PN can be analyzed in unlabeled and largely heterozygous background $\left(\mathrm{tko}^{3 /+}\right)$ by using MARCM system. Progressive phenotype are seen in tko $\mathrm{o}^{3 / 3}$ dendrites, but not in wild type (d$f^{\prime}$ and data not shown). Axonal projections are stable even in 30 days old (a-c and data not shown). Note that dendritic terminal elaborations are sparser in the glomerulus $\left(e^{\prime}, f^{\prime}\right)$, and are seen outside the antennal lobe (arrow in $f^{\prime}$ ). See Chihara et al. (2007) for more details.

etal organization are functionally interrelated.

\section{Mitochondrial dysfunction and neurological diseases}

As described above, mitochondrial distribution and activity are key factors for neuronal morphogenesis, plasticity, and function. In fact, mitochondrial dysfunction is tightly associated with certain inherited human neurological diseases. Mutations in mitochondrial tRNA genes (in the mitochondrial genome) and mitochondrial tRNA synthetase genes (in the nuclear genome) are associated with various neuropathies (Chan, 2006). In addition, OPA1 and mitofusin, which are regulators of mitochondrial morphology, are associated with heredity optic atrophy (Delettre et al., 2002) and Charcot-Marie-Tooth neuropathy type 2A (Zuchner et al., 2004), respectively. Furthermore, mitochondrial functions are directly or indirectly impaired in Parkinson's disease (Greene et al., 2003), Amyotrophic Lateral Sclerosis (ALS) (Liu et al., 2004, Pasinelli et al., 2004), and Down syndrome (Chang and Min, 2005), implying that mitochondria are very important for neurological functioning in development and in adulthood. For comprehensive reviews of the roles of mitochondrial dysfunction in disease, see, for example, Chan (2006) and Kujoth et al. (2005).

\section{Concluding remarks}

The critical importance of intracellular organelles is evident for all eukaryotic cells, including neurons. Recent studies using advanced genetic and microscopic techniques have begun to reveal the essential and specialized roles of each organelle in neuronal morphogenesis and function. Further studies should elucidate the regulatory mechanisms of the intracellular distribution, morphology, and activity of each organelle in each type of neuron. Ideally, analyses carried out in vitro and in vivo will lead to a description of how each organelle contributes to neuronal morphogenesis and function, independently and/or in concert with other organelles.

\section{Note added in proof}

Satoh et al. (2008) and Zheng et al. (2008) have recently reported the possible function of early endosome in the dendritic branching of Drosophila neurons.

\section{Acknowledgements}

We apologize to colleagues whose work could not be cited owing to space limitations. This work was supported in part by grants from the Japanese Ministry of Education, Science, Sports, Culture and Technology, and Core Research for Evolutional Science and Technology (CREST) to T.C. and M.M. In addition, this work was supported in part by Grant-in-Aid for Scientific Research on Priority Areas - molecular brain science-and dynamics of extracellular environments - from MEXT, the Sumitomo Foundation and the Nakajima Foundation to T.C., and Grant-in-Aid for Scientific Research on Priority Areas -Research on Pathomechanisms of Brain Disorders- from MEXT, a RIKEN Bioarchitect Research Grant, the TORA Y Science Foundation, the Cell Science Research Foundation, Naito Foundation and the Astellas Foundation for Research on Metabolic Disorders to M.M.

\section{Reference}

BROWN, T. C., CORREIA, S. S., PETROK, C. N. and ESTEBAN, J. A. (2007). Functional compartmentalization of endosomal trafficking for the synaptic delivery 
of AMPA receptors during long-term potentiation. J Neurosci27: 13311-13315.

CHADA, S. R. and HOLLENBECK, P. J. (2003). Mitochondrial movement and positioning in axons: the role of growth factor signaling. J Exp Bio/206: 19851992.

CHADA, S. R. and HOLLENBECK, P. J. (2004). Nerve growth factor signaling regulates motility and docking of axonal mitochondria. Curr Bio/14: 1272-1276.

CHAN, D. C. (2006). Mitochondria: dynamic organelles in disease, aging, and development. Cel/125: 1241-1252.

CHANG, K. T. and MIN, K. T. (2005). Drosophila melanogaster homolog of Down syndrome critical region 1 is critical for mitochondrial function. Nat Neurosci 8: $1577-1585$.

CHIHARA, T., LUGINBUHL, D. and LUO, L. (2007). Cytoplasmic and mitochondrial protein translation in axonal and dendritic terminal arborization. Nat Neurosci 10: 828-837.

COONEY, J. R., HURLBURT, J. L., SELIG, D. K., HARRIS, K. M. and FIALA, J. C. (2002). Endosomal compartments serve multiple hippocampal dendritic spines from a widespread rather than a local store of recycling membrane. $J$ Neurosci 22: 2215-2224.

CRAIG, A. M., WYBORSKI, R. J. and BANKER, G. (1995). Preferential addition of newly synthesized membrane protein at axonal growth cones. Nature 375 : 592-594.

DELETTRE, C., LENAERS, G., PELLOQUIN, L., BELENGUER, P. and HAMEL, C. P. (2002). OPA1 (Kjer type) dominant optic atrophy: a novel mitochondrial disease. Mol Genet Metab 75: 97-107.

DICKSON, B. J. (2002). Molecular mechanisms of axon guidance. Science 298: 195919-64.

GLATER, E. E., MEGEATH, L. J., STOWERS, R. S. and SCHWARZ, T. L. (2006). Axonal transport of mitochondria requires milton to recruit kinesin heavy chain and is light chain independent. $J$ Cel/ Bio/ 173: 545-557.

GREENE, J. C., WHITWORTH, A. J., KUO, I., ANDREWS, L. A., FEANY, M. B. and PALLANCK, L. J. (2003). Mitochondrial pathology and apoptotic muscle degeneration in Drosophila parkin mutants. Proc Natl Acad Sci USA 100: 4078-083.

GUO, X., MACLEOD, G. T., WELLINGTON, A., HU, F., PANCHUMARTHI, S., SCHOENFIELD, M., MARIN, L., CHARLTON, M. P., ATWOOD, H. L. and ZINSMAIER, K. E. (2005). The GTPase dMiro Is Required for Axonal Transport of Mitochondria to Drosophila Synapses. Neuron 47: 379-393.

HIGGINBOTHAM, H. R. and GLEESON, J. G. (2007). The centrosome in neuronal development. Trends Neurosci30: 276-283.

HISATA, S., SAKISAKA, T., BABA, T., YAMADA, T., AOKI, K., MATSUDA, M. and TAKAI, Y. (2007). Rap1-PDZ-GEF1 interacts with a neurotrophin receptor at late endosomes, leading to sustained activation of Rap1 and ERK and neurite outgrowth. J Cel/ Bio/178: 843-860.

HORTON, A. C. and EHLERS, M. D. (2003a). Dual modes of endoplasmic reticulum-to-Golgi transport in dendrites revealed by live-cell imaging. $J$ Neurosci23: 6188-6199.

HORTON, A. C. and EHLERS, M. D. (2003b). Neuronal polarity and trafficking. Neuron 40: 277-295.

HORTON, A. C., RACZ, B., MONSON, E. E., LIN, A. L., WEINBERG, R. J. and EHLERS, M. D. (2005). Polarized secretory trafficking directs cargo for asymmetric dendrite growth and morphogenesis. Neuron 48: 757-771.

JEFFERIS, G. S., MARIN, E. C., STOCKER, R. F. and LUO, L. (2001). Target neuron prespecification in the olfactory map of Drosophila. Nature 414: 204208.

KElEMAN, K., RAJAgopalan, S., Cleppien, D., TEIS, D., PAIHA, K., HUBER, L. A., TECHNAU, G. M. and DICKSON, B. J. (2002). Comm sorts robo to control axon guidance at the Drosophila midline. Cel/110: 415-427.

KELEMAN, K., RIBEIRO, C. and DICKSON, B. J. (2005). Comm function in commissural axon guidance: cell-autonomous sorting of Robo in vivo. Nat Neurosci 8: 156-163.

KENNEDY, M. J. and EHLERS, M. D. (2006). Organelles and trafficking machinery for postsynaptic plasticity. Annu Rev Neurosci 29: 325-362.

KUJOTH, G. C., HIONA, A., PUGH, T. D., SOMEYA, S., PANZER, K., WOHLGEMUTH, S. E., HOFER, T., SEO, A. Y., SULLIVAN, R., JOBLING, W. A. etal. (2005). Mitochondrial DNA mutations, oxidative stress, and apoptosis in mammalian aging. Science 309: 481-484.

LEE, T. and LUO, L. (1999). Mosaic analysis with a repressible cell marker for studies of gene function in neuronal morphogenesis. Neuron 22: 451-461.

LI, Z., OKAMOTO, K., HAYASHI, Y. and SHENG, M. (2004). The importance of dendritic mitochondria in the morphogenesis and plasticity of spines and synapses. Cel/119: 873-887.

LIU, J., LAMB, D., CHOU, M. M., LIU, Y. J. and LI, G. (2007). Nerve growth factormediated neurite outgrowth via regulation of Rab5. Mol Biol Cel/ 18: 13751384.

LIU, J., LILLO, C., JONSSON, P. A., VANDE VELDE, C., WARD, C. M., MILLER, T. M., SUBRAMANIAM, J. R., ROTHSTEIN, J. D., MARKLUND, S., ANDERSEN, P. M. et al. (2004). Toxicity of familial ALS-linked SOD1 mutants from selective recruitment to spinal mitochondria. Neuron 43: 5-17.

MAXFIELD, F. R. and MCGRAW, T. E. (2004). Endocytic recycling. Nat Rev Mol Cel/ Bio/5: 121-32.

MIYAWAKI, A. (2005). Innovations in the Imaging of Brain Functions using Fluorescent Proteins. Neuron 48: 189-199.

MOISES, T., DREIER, A., FLOHR, S., ESSER, M., BRAUERS, E., REISS, K., MERKEN, D., WEIS, J. and KRUTTGEN, A. (2007). Tracking TrkA's traffiking: NGF receptor trafficking controls NGF receptor signaling. Mo/ Neurobio/35: 151-159.

MORRIS, R. L. and HOLLENBECK, P. J. (1993). The regulation of bidirectional mitochondrial transport is coordinated with axonal outgrowth. J Cel/ Sci104: 917-927.

OVERLY, C. C. and HOLLENBECK, P. J. (1996). Dynamic organization of endocytic pathways in axons of cultured sympathetic neurons. JNeurosci16: 6056-6064.

OVERLY, C. C., RIEFF, H. I. and HOLLENBECK, P. J. (1996). Organelle motility and metabolism in axons vs dendrites of cultured hippocampal neurons. $J$ Cell Sci 109: 971-980.

PARK, M., PENICK, E. C., EDWARDS, J. G., KAUER, J. A. and EHLERS, M. D. (2004). Recycling endosomes supply AMPA receptors for LTP. Science 305: 1972-1975.

PARK, M., SALGADO, J. M., OSTROFF, L., HELTON, T. D., ROBINSON, C. G., HARRIS, K. M. and EHLERS, M. D. (2006). Plasticity-induced growth of dendritic spines by exocytic trafficking from recycling endosomes. Neuron52: 817-830.

PASINELLI, P., BELFORD, M. E., LENNON, N., BACSKAI, B. J., HYMAN, B. T., TROTTI, D. and BROWN. (2004). Amyotrophic lateral sclerosis-associated SOD1 mutant proteins bind and aggregate with $\mathrm{Bcl}-2$ in spinal cord mitochondria. Neuron 43: 19-30.

PREKERIS, R., FOLETTI, D. L. and SCHELLER, R. H. (1999). Dynamics of tubulovesicular recycling endosomes in hppocampal neurons. JNeurosci19: 10324-10337

RODRIGUEZ-BOULAN, E., KREITZER, G. and MUSCH, A. (2005). Organization of vesicular trafficking in epithelia. Nat Rev Mol Cell Biol6: 233-247.

SATOH, D., SATO, D., TSUYAMA, T., SAITO, M., OHKURA, H., ROLLS, M. M., ISHIKAWA, F. and UEMURA, T. (2008). Spatial control of branching within dendritic arbors by dynein-dependent transport of Rab5-endosomes. Nat Cell Biol 10: 1164-1171.

SCHMIDT, M. R. and HAUCKE, V. (2007). Recycling endosomes in neuronal membrane traffic. Biol Cel/99: 333-342.

SHIRANE, M. and NAKAYAMA, K. I. (2006). Protrudin induces neurite formation by directional membrane trafficking. Science 314: 818-8121.

STEWARD, O. and SCHUMAN, E. M. (2003). Compartmentalized synthesis and degradation of proteins in neurons. Neuron 40: 347-59.

STOWERS, R. S., MEGEATH, L. J., GORSKA-ANDRZEJAK, J., MEINERTZHAGEN, I. A. and SCHWARZ, T. L. (2002). Axonal transport of mitochondria to synapses depends on milton, a novel Drosophila protein. Neuron 36: 1063-1077.

SWEENEY, N. T., BRENMAN, J. E., JAN, Y. N. and GAO, F. B. (2006). The coiled-coil protein shrub controls neuronal morphogenesis in Drosophila. Curr Bio/ 16: 1006-11.

TERASAKI, M., SLATER, N. T., FEIN, A., SCHMIDEK, A. and REESE, T. S. (1994). Continuous network of endoplasmic reticulum in cerebellar Purkinje 
neurons. Proc Nat/ Acad Sci USA 91: 7510-7514.

TORRE, E. R. and STEWARD, O. (1996). Protein synthesis within dendrites: glycosylation of newly synthesized proteins in dendrites of hippocampal neurons in culture. $J$ Neurosci 16: 5967-5978.

TOJIMA, T., AKIYAMA, H., ITOFUSA, R., LI, Y., KATAYAMA, H., MIYAWAKI, A. AND KAMIGUCHI, H. (2007). Attractive axon guidance involves asymmetric membrane transport and exocytosis in the growth cones. Nat Neurosci 10: 58-66.

VENKEN, K. J. and BELLEN, H. J. (2005). Emerging technologies for gene manipulation in Drosophila melanogaster. Nat Rev Genet 6: 167-178.

VERSTREKEN, P., LY, C. V., VENKEN, K. J., KOH, T. W., ZHOU, Y. and BELLEN, H. J. (2005). Synaptic mitochondria are critical for mobilization of reserve pool vesicles at Drosophila neuromuscular junctions. Neuron 47:
365-378.

YE, B., ZHANG, Y., SONG, W., YOUNGER, S. H., JAN, L. Y. and JAN, Y. N. (2007). Growing dendrites and axons differ in their reliance on the secretory pathway. Cel/130: 717-729.

ZHENG, Y., WILDONGER, J., YE, B., ZHANG, Y., KITA, A., YOUNGER, S.H., ZIMMERMAN, S., JAN, L.Y. and JAN, Y.N. (2008). Dynein is required for polarized dendritic transport and uniform microtubule orientation in axons. Nat Cell Bio/ 10: 1172-1180.

ZUCHNER, S., MERSIYANOVA, I. V., MUGLIA, M., BISSAR-TADMOURI, N., ROCHELLE, J., DADALI, E. L., ZAPPIA, M., NELIS, E., PATITUCCI, A., SENDEREK, J. etal. (2004). Mutations in the mitochondrial GTPase mitofusin 2 cause Charcot-Marie-Tooth neuropathy type 2A. Nat Genet 36: 449-451.

\section{Further Related Reading, published previously in the Int. J. Dev. Biol.}

See our recent Special Issue Fertilization, in honor of David L. Garbers and edited by Paul M. Wassarman and Victor D. Vacquier at: http://www.ijdb.ehu.es/web/contents.php?vol=52\&issue=5-6

Neurotrophic regulation of retinal ganglion cell synaptic connectivity: from axons and dendrites to synapses

Susana Cohen-Cory and Barbara Lom

Int. J. Dev. Biol. (2004) 48: 947-956

Endocytosis and transcytosis in growing astrocytes in primary culture. Possible implications in neural development.

L Megias, C Guerri, E Fornas, I Azorin, E Bendala, M Sancho-Tello, J M Durán, M Tomás, M J Gomez-Lechon and J Renau-Piqueras

Int. J. Dev. Biol. (2000) 44: 209-221

Analysis of the endocytic-lysosomal system (vacuolar apparatus) in astrocytes during proliferation and differentiation in primary culture.

L Lindo, F J Iborra, I Azorin, C Guerri and J Renau-Piqueras

Int. J. Dev. Biol. (1993) 37: 565-572

Organelle distribution in chick neuroepithelial cells: effects of colchicine and cytochalasin B.

J G Fernández Alvarez, P Paz and C A Chamorro

2006 ISI **Impact Factor $=3.577^{* *}$

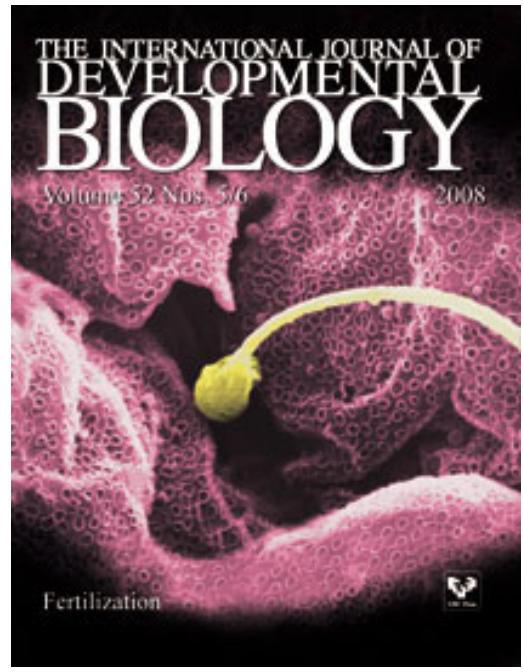

Int. J. Dev. Biol. (1989) 33: 317-323 